\title{
Diagnostics of Air-Breathing Electric Thrusters
}

\author{
Constantinos Stavrinidis', José Gonzalez del Amo', Chloe Berenguer², \\ Konstantinos Katsonis ${ }^{2}$ \\ ${ }^{1}$ ESTEC, European Space Agency, Noordwijk, The Netherlands \\ ${ }^{2}$ DEDALOS Ltd., Thessaloniki, Greece \\ Email: katsonis.dedalos@gmail.com
}

Received 5 June 2015; accepted 15 October 2015; published 22 October 2015

\begin{abstract}
Air-Breathing Electric Thrusters (ABET) diagnostics is addressed, with on-ground prototypes in mind. It is based on detailed volume averaged Global Models and focuses on emission spectroscopy. Notably, the obtained optical emission spectroscopy diagnostics tools give important information about the thruster propellant constitution and also the ionization degree of each constituent. This allows for trade-off between various prototypes on the basis of their characterization and optimization.
\end{abstract}

Keywords

Diagnostics, Low Earth Orbit, Electric Thrusters, Air-Breathing, Spectroscopy

\section{Introduction}

Diagnostics constitutes an important tool for electric thruster technology development and, further, for thruster in-flight monitoring. Especially for a disruptive technology as is this of air-breathing, detailed diagnostics taking into account the propellant constitution becomes necessary, both in the thruster development phase and for optimization of its functioning.

Contemporary extensive on-ground experimental studies, needed for trade-off between various prototypes of Air-Breathing Electric Thrusters (ABET), led to development of performing diagnostics tools. Among them, the specificity of the considered thruster propellants suggests an extensive use of Optical Emission Spectroscopy (OES) to give information about the constitution of the propellant and the main species densities, in relation with important parameters as electron density $\left(n_{e}\right)$, electron temperature $\left(T_{e}\right)$ etc. The fundamental parameter of ionization degree directly related with thrust is also evaluated. OES is used to characterize various experimental devices creating on-ground conditions similar to those of the Low Earth Orbit (LEO) environment, including those generating hyperthermal beams, mainly of oxygen and nitrogen species.

A convenient model is necessary as a theoretical support of OES, either of the Collisional-Radiative (C-R) type if the plasma dimensions are quite extended and without fundamental importance, or of the Global Model (GM) type, the latter giving additional information about how the available energy is spent within the device. Dedicated GMs lately made available constitute a powerful modeling support meant for thrusters and related 
devices [1] [2]. Thus, they contribute considerably to the development of the ABET disruptive technology [3]. GM applications include thruster feeding by the Atmospheric Remnants (AtR) in LEO conditions at about 200 $\mathrm{km}$ [4]. Ar needs often to be considered, because, together with He, it is the most common species in this region after $\mathrm{O}, \mathrm{N}_{2}, \mathrm{O}_{2}$ and $\mathrm{N}$ ones and also because it is currently used as carrier gas. Description of OES diagnostics of an Ar-fed helicon thruster may be found in [5].

Especially for AtR feeding, a Four Components Global Model (4CGM) [3] was developed at DEDALOS Ltd., addressing initially the four main components of the atmosphere composition in about $200 \mathrm{~km}$ altitude. Included in this model is detailed structure of N I, II and of O I, II. Besides its modeling application, 4CGM also constitutes a basis for OES diagnostics of plasmas of interest to air-breathing technology. We are looking forward to apply the non-intrusive OES supported by 4CGM in diagnosing mini-Hall and Helicon type ABET devices, by using experimental facilities available at the ESA Propulsion Laboratory (EPL) [6] of the European Space Agency (ESA), ESTEC, as soon as the necessary devices will be installed and in compliance with the availability schedule. This model could be also used to characterize AtR collectors and propellant reservoirs. Moreover, also a five component GM (5CArGM) encompassing Ar, was lately developed at DEDALOS Ltd. It includes detailed atomic structure leading to low order Ar I, II, III spectra [3].

Propellant composition analysis was obtained on the basis of the main present species theoretical spectra. Visible and UV spectral regions were addressed, where intense lines from N I, II, O I, II and Ar I, II, III excited levels transitions have been observed. Main emission lines of ionized species lie in the UV region, while low lying transitory levels give the most intense resonant lines in the VUV region.

After this introduction, we review in Section 2 standard diagnostics used in on-ground ABET prototypes. Support for OES by detailed models of C-R and of GM type is the subject of Section 3. Section 4 presents important effects revealed by GM studies of low pressure ABETs fed by AtR and $\mathrm{O}_{2}$ in case of absorbed power variation. In Section 5 we address theoretical atomic spectra corresponding to AtR propellant. Main conclusions of the present work are presented in Section 6.

\section{On-Ground ABET Diagnostics}

Standard diagnostics devices used currently on-ground for ABET thruster plasmas characterization can be summarized as follows:

- Mass flow controller, to quantify each of the provided component.

- Faraday cup, to measure the ion current density.

- Langmuir probe, to measure the plasma potential.

- Retarding Potential Analyzer (RPA), to perform ion energy analysis.

- Inverted-Pendulum Thrust Stand, to measure the thruster performance.

Details about installation and use of the aforementioned diagnostics devices can be found elsewhere, see e.g. [7] and references therein. Such devices constitute basic tools necessary for Electric Thruster (ET) and beam diagnostics, therefore, they have been made available at EPL. They have been taken into consideration when EPL has been ISO17025 certified for measurements in the following ranges:

-Force; $1 \mu \mathrm{N}$ to $500 \mathrm{mN}$.

-Electrical power; $0.1 \mathrm{~W}$ to $2 \mathrm{~kW}$.

-Mass flow; $0.1 \mathrm{mg} / \mathrm{s}$ to $300 \mathrm{mg} / \mathrm{s}$.

Among other applications, the diagnostics devices available at EPL allowed recently for experimental validation of an integrated mini-RIT subsystem meant for LEO applications [8].

Description of experimental facilities meant for hyperthermal beam diagnostics has been early made available in a review [9] describing various ways of fast beam production. Ref. [9] was also referring to diagnostics tools, including references to original papers published up to 1995. A brief overview of the most basic methods that are used for characterization of the atomic oxygen beams is given in [10], where the related literature up to 2003 is also to be found. As reported in [10], atomic oxygen beam energy measurements are most commonly made by Time-of-Flight (ToF) method. The latter use often choppers for modulation and mass spectrometers in case of seeded beams. Mass spectrometers are also used to determine the dissociation degree of the beam. Various methods to measure the beam flux are reported in [10], but the most commonly used nowadays is the measurement based on the polyimide film erosion technique.

Specific diagnostics methods and devices used currently in ABET and oxygen atomic beams technology have 
been described in [11], with emphasis to LEO satellites propulsion applications. Review of diagnostics methods in [11] was often made in conjunction with a summary description of the devices in which they were applied. It appears that Laser Absorption Spectroscopy (LAS) has been extensively used. The important OES diagnostics and the GMs which are necessary as a theoretical support of OES [3] are addressed separately in [11]. More generally, GMs constitute a powerful tool in supporting selection among In Situ Resource Utilization (ISRU) ETs, allowing also for their modeling and optimization.

VUV radiation from atoms and ions (roughly from $100 \mathrm{~nm}$ to $200 \mathrm{~nm}$ ) and notably from the oxygen species has important surface effects. Due also to its specific experimental requirements, VUV radiation is diagnosed separately. Typical emission spectral lines for oxygen are observed at about $130.4 \mathrm{~nm}$ (resonant $2 p$-3s VUV line), $394.7 \mathrm{~nm}, 436.8 \mathrm{~nm}(3 \mathrm{~s}-4 p)$, $777.2 \mathrm{~nm}$ and $844.6 \mathrm{~nm}(3 s-3 p)$.

\section{Detailed Collisional-Radiative and Global Models Supporting OES}

A conveniently adapted model is necessary as an OES theoretical support, either of the C-R type, if the plasma dimensions are quite extended and hence without fundamental importance, or of the GM type, the latter giving additional information about how the available energy is spent within the device. Optical diagnostics is then relying to the GM results [4], or at least to those obtained by (C-R) models [12]. The advantage of using GM instead of C-R models in ET diagnostics is that the former results to Functioning Diagrams (FD) [3] [13] giving an overview of the ET plasma, including $T_{e}$ and $n_{e}$ values. Whenever direct interactions within various excited states can be considered as being negligible, spectroscopic measurements can test the internal consistency of the GM by means of comparison with C-R ones. Each FD point corresponds to a spectrum snapshot which reveals the theoretical characterization of the plasma situation. Inversely, it is possible to assess the plasma conditions for known values of dimensions and feed, comparing acquired spectra with a collection of calculated ones.

Coronal models have been occasionally used as an approximation, although, because in this case ionization comes only from the ground level and also only recombination to the ground level is considered, the shape of the theoretical multiplets lines becomes independent of $T_{\mathrm{e}}$. Dedicated GMs lately made available [13], constitute a modeling support meant for thrusters and related devices [1], allowing for further development of the ABET disruptive technology [3]. GM application encompasses here thruster feeding by AtR under LEO conditions of about $200 \mathrm{~km} \mathrm{[4]} \mathrm{[14].} \mathrm{Together} \mathrm{with} \mathrm{O}, \mathrm{N}_{2}, \mathrm{O}_{2}$ and $\mathrm{N}$, the most common species in this region, $\mathrm{Ar}$ is also to be considered, because is currently used as carrier gas. Note that OES diagnostics details of an Ar-fed helicon thruster may be found in [5]. In order to constitute a basis for OES diagnostics of plasmas of interest to airbreathing technology, 4 CGM [4], addressing initially the four main components must include detailed structure of N I, II and O I, II species, because, as we will see in Section 5 the second order spectra are very present.

Due to the frequent use of Ar as carrier gas in a multitude of devices of interest to LEO technology, we developed the aforementioned five initial component GM, by adding Ar to the initial constituents of 4CGM. Because a considerable number of Ar related studies exists, this model, 5CArGM, allows for standard OES in extended $T_{e}$ and $n_{e}$ domains, once even a small amount of Ar and/or of its lowly ionized species is present. Theoretical spectra of Ar I to III species have been calculated with 5CArGM, corroborating our previous C-R model results [15]. A typical example of 5CArGM application was given in [3].

\section{Effects of the Absorbed Power Variation in Low Pressure ABETs}

Effects of a few mTorr pressure variation of the ABET propellants have been addressed in [16] by means of 4CGM. Absorbed power effects were addressed in [11]. Typical power variation results are illustrating here the GM contribution to OES diagnostics. They address power variation from a low value of $200 \mathrm{~W}$ up to $1 \mathrm{~kW}$, in case of a feed by the $180 \mathrm{~km}$ altitude AtR mixture. Results obtained by 4CGM are shown in Figure 1. They are compared with those obtained in case of an $\mathrm{O}_{2}$ feeding, shown in Figure 2. For this comparison, a typical pressure of $1 \mathrm{mTorr}$ has been selected, for a feed of $40 \mathrm{sccm}$. Figure 1 and Figure 2 constitute a power depending version of the standard Plasma Component Composition (PCC) diagrams [4] which give the propellant constitution as a function of the absorbed power $\left(\mathrm{P}_{\mathrm{abs}}\right)$.

Component variations observed in Figure 1 and Figure 2 are comparable to those previously presented in Figure 10 and Figure 11 of [3]. However, the AtR feeding is here double than in the case of [3]. We observe differences between results pertaining to $20 \mathrm{sccm}$ and to $40 \mathrm{sccm}$, especially for low absorbed power values, as the power available in this case is not enough to both sufficiently dissociate the $\mathrm{N}_{2}$ and $\mathrm{O}_{2}$ molecules and quite 


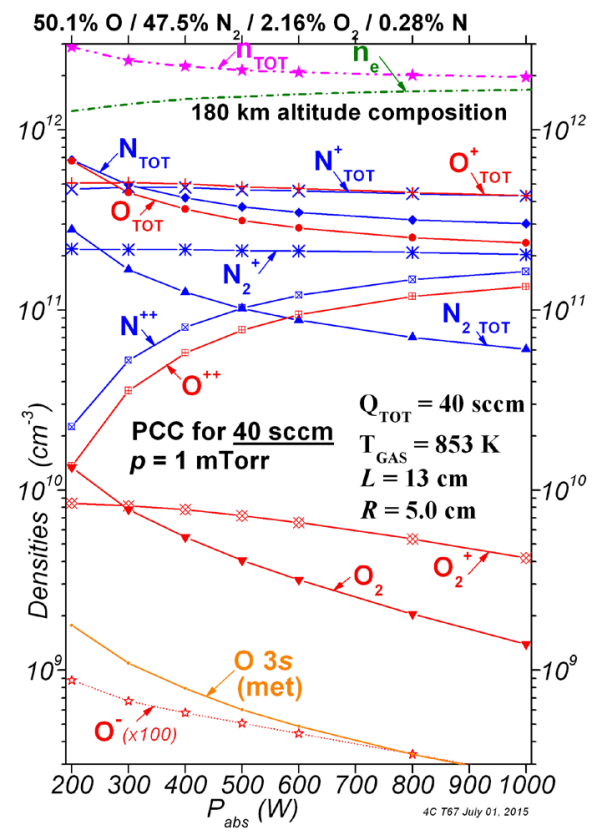

Figure 1. Power-depending $1 \mathrm{mTorr}$ PCC for $40 \mathrm{sccm}$ atmospheric mixture feeding. Form factor of $L=13 \mathrm{~cm}, R=5.0 \mathrm{~cm}$.

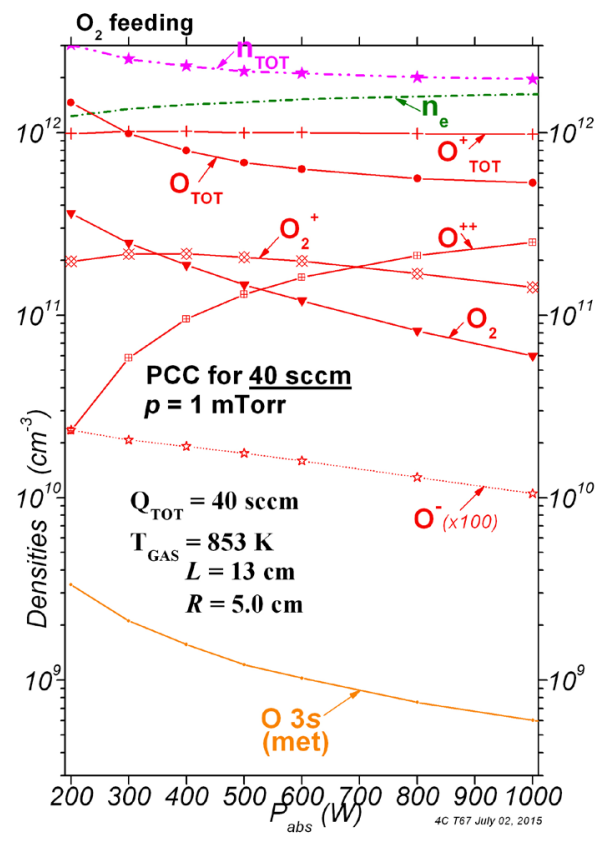

Figure 2. Power-depending $1 \mathrm{mTorr}$ PCC for $40 \mathrm{sccm} \mathrm{O}_{2}$ feeding. Form factor of $L=13 \mathrm{~cm}, R=5.0 \mathrm{~cm}$.

ionize the thruster plasma components. Moreover, results for AtR mixture and for $\mathrm{O}_{2}$ feeds are quite different, explaining why pure atomic beams cannot satisfactorily describe the physico-chemical phenomena belonging to a similar AtR feed.

Once experimental atomic spectra become available, their comparison with theoretical ones provided by GM calculations as those resulting e.g. to Figure 1, gives the values corresponding to basic plasma parameters. It is to be noted that, in agreement with the calculated PCC shown in Figure 1 indicating important presence of various molecules, intense molecular spectra are also generally present in the obtained spectral acquisitions. 
In [16] various types of ABET prototypes were considered, following an increasing effort of air breathing technology development. Overall theoretical feasibility of ram-type Electric Propulsion (RAM-EP) has been studied previously by an ad hoc ESA interdisciplinary team under the responsibility of J. Gonzalez del Amo and D. Di Cara, as part of the Concurrent Design Facility (CDF) [17]. This study included also an overview of the required technology development for ABET. Recent on-ground experimental devices of interest to ABET are often using $\mathrm{N}_{2}$ and/or air feed as is the case with Helicon Hall Thruster (HHT) [7], low power radio frequency Helicon Plasma Thrusters (HPT) fed e.g. by argon [18] or laser detonation hyperthermal beam sources [19] addressing atomic oxygen feed. Besides oxygen and/or nitrogen feed, which are the most abundant constituents in LEO environment, propulsion and carrier gases are used in on-ground experiments, aiming to increase O/N speed. These are preferably rare gases, typically Ar which was mentioned previously. Note that inert gases absorb the provided electric power mainly for excitation and for ionization by electron collisions, set aside atomic collisions within various species, a process becoming important in higher pressures. Note that $\mathrm{O}_{2} / \mathrm{Ar}$ mixtures may conveniently simulate LEO environment, as was conjectured lately [20] and corroborated by theoretical GM results recently presented in [3].

\section{Theoretical Atomic Spectra}

In this section, we address typical theoretical atomic spectra obtained by 4 CGM. As reported previously, these are necessary for a comparison with experimental ones, in order to obtain characterization by OES diagnostics in presence e.g. of oxygen and nitrogen mixtures feeding. Spectra vary as a function of the absorbed power. Two typical spectra are presented and discussed in the following. Typical conditions and mixture composition are those of Figure 1. Pressure is fixed at $1 \mathrm{mTorr}$ and flow rate is of $40 \mathrm{sccm}$. Dimensions are of $L=13 \mathrm{~cm}$ and $R$ $=5 \mathrm{~cm}$ with gas temperature of $853 \mathrm{~K}$.

Theoretical spectrum illustrated in Figure 3 corresponds to AtR feed in conditions pertaining to the PCC presented in Figure 1. It has been obtained by our 4CGM for a typical plasma thruster fed with AtR and $200 \mathrm{~W}$ absorbed power. In this figure, lines of neutral atomic species O I (red lines), N I (blue lines) and ionic species O II (red dashed lines), N II (blue dashed lines) are presented. We observe that both neutral and ionized species result to quite intense lines. Also, we see that in the visible part of the spectrum, among the most intense lines are these of O I $(\lambda=777 \mathrm{~nm})$ followed by N I $(\lambda=745 \mathrm{~nm})$.

Figure 4 shows details of the previous Figure 3 obtained by 4CGM in the UV, visible and near infrared regions, concerning exclusively the main theoretical lines of O I (red lines) and N I (blue lines) species. Some of these lines belong to averaged levels. Lines O I $(\lambda=1129 \mathrm{~nm}$ and $(\lambda=1130 \mathrm{~nm}$ are almost blended in the chosen wavelength representation.

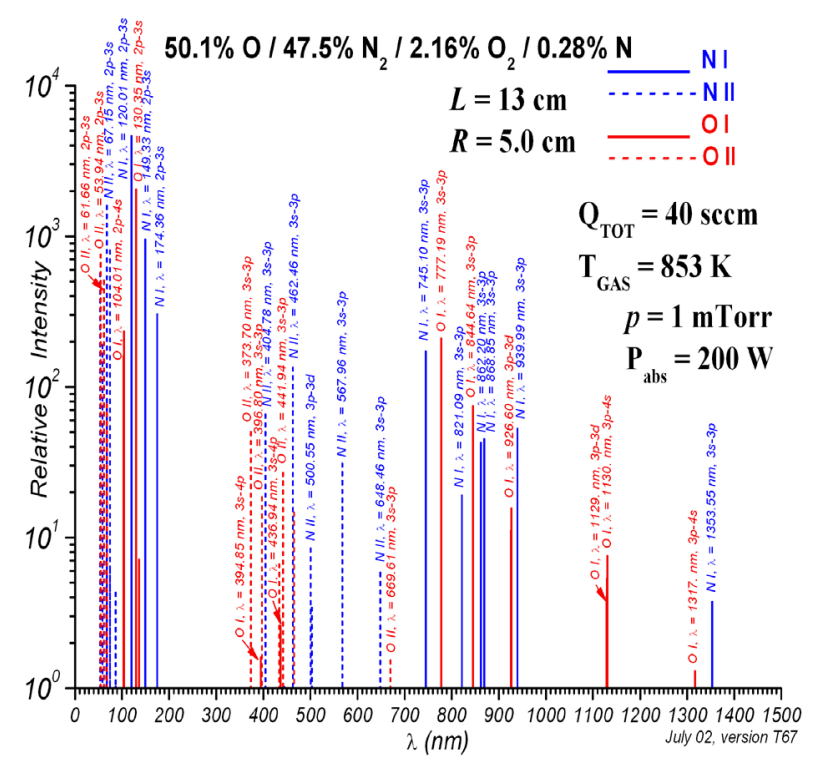

Figure 3. Theoretical spectrum of a plasma thruster fed with AtR. Pressure is of 1 mTorr and absorbed power is of $200 \mathrm{~W}$. 


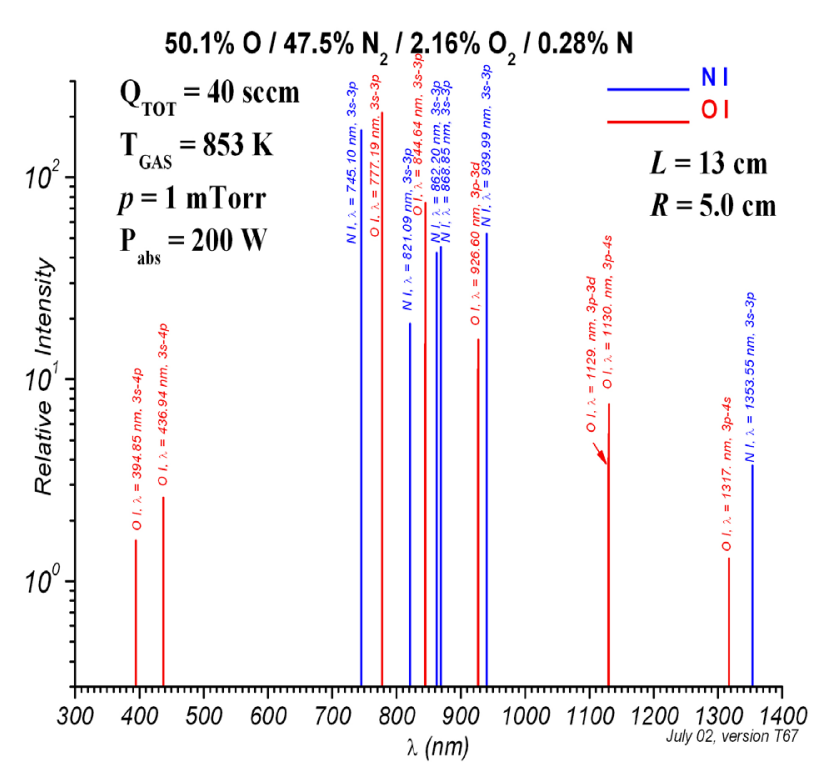

Figure 4. Main neutral species lines from Figure 3 in the region from $300 \mathrm{~nm}$ to $1400 \mathrm{~nm}$.

\section{Conclusion}

Specific diagnostics methods used currently in ABET and oxygen atomic beams technology have been summarily addressed, with emphasis to LEO satellites propulsion applications. OES diagnostics helping in prototypes trade-off has been made, facilitating ABET selection. The fundamental non-intrusive OES diagnostics and the GMs which are necessary as a theoretical support of it were addressed. Expected ABET characteristics variation has been described as a function of the absorbed power. Obtained theoretical spectra illustrate the possibility of using detailed global models to diagnose plasma thrusters using OES, notably when fed by gas mixtures which are of interest to ABET propulsion. It is then illustrated that GMs constitute a powerful tool in supporting selection of ISRU ETs, allowing both for their modeling and for their optimization.

\section{Acknowledgements}

Ch. Berenguer and K. Katsonis thank ESA/ESTEC/TEC-MPE for its support.

\section{References}

[1] Berenguer, Ch. and Katsonis, K. (2012) Plasma Reactors and Plasma Thrusters Modeling by Ar Complete Global Models. Int. J. Aerospace Eng., 2012, ID 740869. http://dx.doi.org/10.1155/2012/740869

[2] Berenguer, Ch. and Katsonis, K. (2014) Detailed Global Modeling of Low Pressure Nitrogen Plasmas. ESA 6th RHTG Workshop, St. Andrews, November 2014.

[3] Katsonis, K., Berenguer, Ch. and Gonzalez del Amo, J. (2015) Characterization of Air Breathing Plasma Thrusters Fuelled by Atmospheric Mixtures Encountered in Earth Atmosphere at an Altitude of About $200 \mathrm{~km}$. 34th IEPC Conference, Kobe, July 2015, IEPC-2015-268.

[4] Katsonis, K. and Berenguer, Ch. (2014) Properties of the Atmospheric Mixtures Prevailing around 200 km Altitude in Conditions of Low Absorbed Power and Pressure. ESA 6th RHTG Workshop, St. Andrews, November 2014.

[5] Katsonis, K., Berenguer, Ch., et al. (2011) Optical Diagnostics of a Low Temperature Argon Thruster. 32nd IEPC Conference, Wiesbaden, September 2011, IEPC-2011-169.

[6] Gonzalez del Amo, J., Saccoccia, G. and Frigot, P.-E. (2009) ESA Propulsion Lab at ESTEC. 31st IEPC Conference, Ann Arbor, IEPC-2009-236.

[7] Shabshelowitz, A. (2013) Study of RF Plasma Technology Applied to Air-Breathing Electric Propulsion. Ph.D. Thesis, University of Michigan, MI.

[8] Di Cara, D., Bulit, A., Gonzalez del Amo, J., Romera, J.A., Leiter, H., Laner, D., et al. (2013) Experimental Validation 
of RIT Micro-Propulsion Subsystem Performance at EPL. 33rd IEPC Conference, Washington DC, October 2013, IEPC-2013-90.

[9] Chutjian, A. and Orient, O.J. (1996) Fast Beam Sources. Experimental Methods of the Physical Sciences, 29, 49. http://dx.doi.org/10.1016/S0076-695X(08)60785-1

[10] Kleiman, J., Iskanderova, Z., Gudimenko, Y. and Horodetsky, S. (2003) Atomic Oxygen Beam Sources: A Critical Overview. 9th Int. Symp. on Materials in a Space Environment, Noordwijk, June 2003, ESA SP-540.

[11] Katsonis, K. and Berenguer, Ch. (2015) Measurement System Identification Report for On-Ground Testing. Technical Note TN-3 for ESA/ESTEC Project No. IPL-PTM/PA/st/541.2014, Assessment of a Neutral Particle Generator, Report DE-2015-03, Thessaloniki, May 2015.

[12] Katsonis, K., Berenguer, Ch., Bonnet, J., Packan, D., Clark, R.E.H., Cornille, M. and Maynard, G. (2007) A Xenon Collisional-Radiative Model for Plasma Thruster Optical Diagnostics and Modeling. 30th IEPC Conference, Florence, September 2007, IEPC-2007-285.

[13] Katsonis, K. and Berenguer, Ch. (2013) Global Modeling of $\mathrm{N}_{2} \mathrm{O}$, Air and $\mathrm{N}_{2}$ Discharges and Applications. Lambert Academic Publishing, Germany.

[14] Katsonis, K., Berenguer, Ch., Gonzalez del Amo, J. and Stavrinidis, C. (2015) Atmospheric Remnants in the Low Earth Orbit Region around 200 km Altitude. CASE 2015 Conference, Suzhou, October 2015.

[15] Katsonis, K., Boisse-Laporte, C., Bonnet, J., Letout, S. and Siskos, A. (2004) C-R Modeling and Spectroscopic Diagnostics of SPT Plasmas. Proceedings of the 4th International Spacecraft Propulsion Conference (ESA SP-555), Chia Laguna, Cagliari, Sardinia, June 2004.

[16] Katsonis, K. and Berenguer, Ch. (2015) Preliminary Design Report Particle Generator, Technical Note TN-2 for ESA/ ESTEC Project No. IPL-PTM/PA/st/541.2014, Assessment of a Neutral Particle Generator, Report DE-2015-02, Thessaloniki, March 2015.

[17] CDF Study Report, Concurrent Design Facility, CDF-55(A), RAM-EP Study Report, January 2007.

[18] Pavarin, D., Lucca Fabris, A., Trezzolani, F., Manente, M., Faenza, M., Ferri, F., Selmo, A., Katsonis, K. and Berenguer, Ch. (2012) Low Power RF Plasma Thruster Experimental Characterization. 48th AIAA Conference, Atlanta, July-August 2012. http://dx.doi.org/10.2514/6.2012-4191

[19] Yoneda, S., Tanaka, K., Nomura, S., Matsui, M., Yamagiwa, Y., Komurasaki, K. and Arakawa, Y. (2011) Generation of High Speed Atomic Oxygen Flows by a Laser Driven Plasma Wind Tunnel. 28th International Symposium on Space Technology and Science, Okinawa, 2011, 2011-r-60p.

[20] Yokota, K., Watanabe, D., Ohira, J. and Tagawa, M. (2014) Multiple-Composition Hyperthermal Atomic Beams Formed by a Laser-Induced Plasma for Planetary Environmental Studies. Phys. Scr., T161, 014035. http://dx.doi.org/10.1088/0031-8949/2014/t161/014035 